\section{'Inc-miRs': functional intron-interrupted miRNA genes}

\author{
Huibin Zhang, ${ }^{1}$ Jay M. Maniar, ${ }^{1}$ \\ and Andrew Z. Fire ${ }^{1,2,3}$ \\ ${ }^{1}$ Department of Genetics, Stanford University, Stanford, \\ California 94305, USA; ${ }^{2}$ Department of Pathology, Stanford \\ University, Stanford, California 94305, USA
}

The discovery of microRNAs (miRNAs) lin-4 and let-7 as temporal regulators in Caenorhabditis elegans led to broader searches for novel miRNAs and their biological roles. Unlike protein-coding genes and some long noncoding RNAs, canonical metazoan miRNAs are not known to contain introns within their genomic precursor sequences. Because the short length of miRNAs complicates a statistically definitive assignment of split genes in RNA sequencing data sets, we took an experimental approach toward testing the compatibility of splicing and functional miRNA biogenesis. To definitively evaluate the possibility that miRNAs could derive from interrupted genes, we constructed intron-interrupted variants of $C$. elegans lin- 4 and assayed for their miRNA-encoding capability and biological activity in the developing organism. Our studies indicate that (1) intron-containing miRNAs (inc-miRs) can be efficiently spliced and processed to produce miRNAs with normal termini, and (2) these miRNAs can be functional in full rescue of developmental phenotypes in null mutants lacking endogenous lin-4. This study provides the first evidence to support the ability of intron-interrupted miRNA precursors to produce functional regulators and identifies an additional modality available for metazoan miRNA production.

Supplemental material is available for this article.

Received April 12, 2011; revised version accepted June 16, 2011.

MicroRNAs (miRNAs) were first discovered with the identification of lin-4 as a regulator of temporal development in Caenorhabditis elegans (Lee et al. 1993; Wightman et al. 1993). Although a handful of other miRNAs have been identified through genetics means (Reinhart et al. 2000; Johnston and Hobert 2003), the number of miRNAs discovered using forward genetics has remained small. The majority of C. elegans miRNA knockouts do not display overt phenotypes, indicating a combination of overlapping phenotypes (partial or complete redundancy) and subtle biological effects in this system (Miska et al. 2007; Alvarez-Saavedra and Horvitz 2010).

[Keywords: C. elegans; intron; lin-4; microRNA; splicing]

${ }^{3}$ Corresponding author.

E-mail afire@stanford.edu.

Article is online at http://www.genesdev.org/cgi/doi/10.1101/gad.2058711.
With the advent of better small RNA capture methods and high-throughput sequencing, thousands of miRNAs have now been identified in a variety of organisms (Ambros and Lee 2004; Pfeffer et al. 2005; Kloosterman et al. 2006; Rajagopalan et al. 2006). Some of the criteria used in novel miRNA annotation include the prediction of a fold-back precursor structure, detection of the partially complementary "star" strand, and phylogenetic conservation of the candidate miRNA (Ambros et al. 2003). Although a large proportion of miRNAs still have unknown functions (Bushati and Cohen 2007), much headway has been made in understanding miRNA biogenesis, as well as identifying the important players in this pathway (Kim et al. 2009).

miRNA genes may be transcribed either from their own promoters, as part of a cluster of miRNA genes, or as part of a host gene (Fig. 1A; Kim et al. 2009). These long transcripts are capped and spliced to form primary miRNAs (pri-miRNAs) in metazoans (Cai et al. 2004; Lee et al. 2004). Pri-miRNAs are processed by Drosha to produce $\sim 70$-nucleotide (nt) stem-loop intermediates, known as precursor miRNAs (pre-miRNAs) (Kim et al. 2009). Dicer then acts on pre-miRNAs to generate $\sim 22-$ nt mature miRNAs (Kim et al. 2009). In the case of mirtrons, premiRNAs are spliced directly out of small introns, therefore bypassing the Drosha processing step (Okamura et al. 2007; Ruby et al. 2007). Furthermore, in cases where premiRNAs of mirtrons are derived from longer introns, the exosome has been found to mediate $3^{\prime}$ end trimming to yield the pre-miRNA (Fig. 1A; Flynt et al. 2010).

Protein-coding genes often require splicing to remove introns that interrupt both coding and noncoding sequences (Soller 2006). Intron removal has also been observed with long noncoding RNAs (Mattick and Makunin 2006). In contrast, metazoan miRNA genes are not known to contain introns within the pre-miRNA or mature miRNA sequences (Fig. 1B). None of the few miRNAs and pre-mRNAs identified through "forward genetics" contains an intron. The bulk of characterized miRNAs, identified first through high-throughput sequencing, also contains no intron interruptions. It should be pointed out that the standard methodology of novel miRNA discovery was not designed to find such intron-interrupted entities, since algorithms that have been used for miRNA annotation apply an initial filter of requiring a perfect match to the relevant genome (LagosQuintana et al. 2001; Lau et al. 2001; Lee and Ambros 2001). It is certainly conceivable that such endogenous intron-containing miRNAs (inc-miRs) may exist; both protein-coding and miRNA genes are transcribed by RNA polymerase II, unlike other noncoding small RNA genes such as tRNAs (Lee et al. 2004). In addition, the studies on the biogenesis of mirtrons and other miRNAs that reside in introns of host genes show that splicing can be integrated into the biogenesis pathway to generate mature miRNAs and, in the case of mirtrons, plays an essential role (Kim and Kim 2007; Okamura et al. 2007; Ruby et al. 2007). Intriguingly, there is one instance in plants (miR444) in which splicing signals close to the loop region of a miRNA precursor are conserved and where splicing could contribute to the production of a canonical Dicer substrate (Sunkar et al. 2005; Lu et al. 2008; Thieme et al. 2011). A computational 


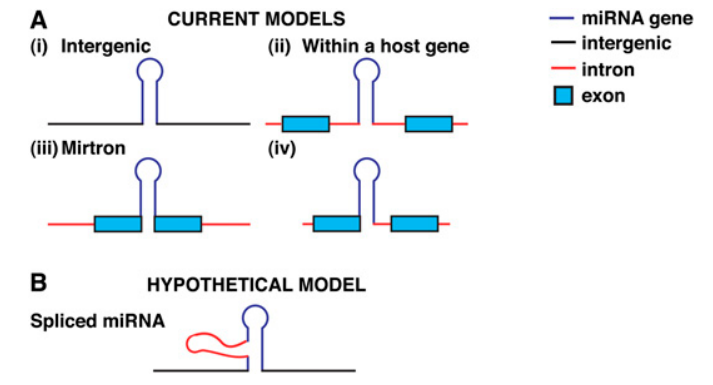

Figure 1. miRNA gene models. $(A)$ Current miRNA gene models. Canonical miRNA genes can be found in intergenic regions as independent transcriptional units (panel i) or in introns of host genes (panel ii), where they may be transcribed independently or as part of host gene transcripts. (Panel iii) Mirtrons are miRNAs that originate from small introns of host genes. Mirtrons bypass Drosha processing as splicing releases the mirtron precursors from the host transcripts. (Panel iv) In cases where the introns are longer than the mirtron precursors, the exosome machinery trims the spliced intron to the appropriate mirtron precursor length. $(B)$ Hypothetical gene model for inc-miRs. The genomic sequence of a hypothetical incmiR gene includes an intron within the inc-miR precursor sequence, unlike canonical miRNA and mirtron precursors. The resulting mature inc-miR would be a spliced product.

search for intron-interrupted miRNA genes in C. elegans small RNA data sets (Maniar and Fire 2011) was carried out, but the short length of these RNAs complicates the establishment of genomic origins. Indeed, by sheer numbers of possibilities for start sites and intron locations, a substantial fraction of random 21-mers is assignable to interrupted genomic segments. Additional inherent ambiguities in post-transcriptional modifications- such as RNA editing (Luciano et al. 2004), 3' nucleotide additions (Li et al. 2005), and mRNA degradation-further complicate any definitive assignment of intron-interrupted miRNA genes based on sequence data alone. These considerations led us to address the possibility of spliced miRNAs more directly, using a defined assay system.

In this study, we created intron-interrupted lin-4 constructs and assayed for their lin-4 rescue activities using the well-characterized lin-4 phenotypic rescue assay in $C$. elegans. The introns in these constructs interrupt the pre-miRNA sequence. The rescue of the lin-4-null phenotype and subsequent high-throughput sequencing and analyses of captured mature lin-4 populations in these transgenic animals indicate that incmiRs can be accurately spliced to produce functional miRNAs efficiently.

\section{Results and Discussion}

In vivo function of intron-containing lin-4 constructs

Rescue of null mutations in the well-characterized $C$. elegans miRNA lin-4 provides a definitive assay for miRNA functionality. This heterochronic miRNA plays an important role in temporal control of development, especially during the L1-to-L2 transition /Chalfie et al. 1981). In animals that lack lin-4, development does not occur in a timely fashion and specific tissues fail to terminally differentiate; lin-4(e912) animals fail to form adult alae, lack a functional vulva, and essentially never lay eggs (Chalfie et al. 1981). We examined the functionality of lin-4 when interrupted by a class II intron and introduced into populations of lin-4-null mutant animals using DNA-mediated transformation.

Twelve intron-containing lin-4 constructs (int_1 to int_12) were made using a synthetic 51-nt intron (Fig. 2A, panel i) previously used in GFP constructs for $C$. elegans expression (Fire et al. 1998). The 695-nt lin-4 genomic sequence used in these constructs (Fig. 2A, panel ii) has been shown to rescue the lin-4-null phenotype (Lee et al. 1993). The 51-nt intron was inserted at different locations within the lin-4 precursor sequence (indicated by arrows in Fig. 2B). These intron-containing lin-4 constructs were introduced into lin-4(e912) animals and the transgenic strains were initially assayed for lin-4 rescue activity by assessing vulva formation at the L4 larval and adult stages.

As shown in Figure 2C, several intron-interrupted constructs showed rescue of vulval development at levels comparable with those seen with the parental (wild-type) plasmid. Different degrees of rescue were observed for the various insertion points tested. To provide more accurate assessments of lin-4 rescue activities, the transgenic animals were categorized into four different phenotypes: (1) egg-laying, (2) protruding vulva (pvl), (3) bursting through a partially formed vulva (bursting), and (4) vulvaless. Both pvl and vulvaless animals retain fertilized eggs and eventually become "bags of worms" when the progeny hatch inside them. Animals with a "bursting vulva" phenotype usually do not survive to produce progeny. As there is some variation in exogenous DNA expression between transgenic lines (due to mosaicism, copy number variation, and transgene silencing), we observed a range of rescue phenotypes associated with vulva formation. By considering all three phenotypic categories that indicated signs of vulva formation (egg-laying, pvl, and bursting) and comparison with transgenic animals expressing a wildtype lin-4 construct (no intron, 48\%), five constructs (int_3, int_5, int_8, int_9, and int_10) showed robust lin-4 rescue activities (ranging from 30\%-73\%) (Fig. 2C). The remaining intron-containing lin-4 constructs had varying limited levels of rescue activity; between $2 \%$ and $17 \%$ of assayed transgenic animals displayed restoration of vulva formation (Fig. 2C). A detailed summary of the percentages of transgenic animals in each phenotypic category for each transgenic line is found in Supplemental Figures 1 and 2.

In addition to vulval formation and egg-laying, a lack of lin-4 also causes a number of other developmental defects, including defects in cuticle patterning (a loss of adult cuticular structures called alae) and whole-animal growth rate (about a half day longer in the time needed to develop from embryo to $\mathrm{L} 4$ at $25^{\circ} \mathrm{C}$ from our observations) (Chalfie et al. 1981). We assayed transgenic animals from lines with robust rescue (int_3, int_5, int_5sm, int_8, and int_10) and found that adult alae were present in these populations $(27 \%-96 \%$, compared with $31 \%$ for parental [wild-type] lin-4 construct). Populations of rescued animals also exhibited rescued growth rates that were comparable with wild-type N2 populations (alleviating the approximately half-day lag observed in lin-4-null mutants); together with the rescue of vulval development and egg-laying, these observations strongly suggest that intron-interrupted lin-4 constructs can provide functionality in developmental progression. 
Splicing is required for rescue activity of intron-containing lin-4 constructs

To test whether splicing was required for mature lin-4 production from intron-interrupted lin-4 constructs, we mutated the splice sites of two intron-interrupted lin-4 constructs (int_3 and int_5) previously shown to have robust lin-4 rescue activities. The synthetic intron, built with the consensus class II intron structure <gt. . ag > (Blumenthal and Steward 1997; Soller 2006), was changed by mutation at the junctions to yield <ag. . .ct $>$ (Fig. 3A). Transgenic animals expressing these mutated introninterrupted lin-4 constructs (int_3sm and int_5sm) were assayed for their lin-4 rescue activities.

As shown in Figure 3B, when compared with rescue percentages from the previous experiments (int_3 and int_5), splice site mutations in construct int_3 prevented effective rescue of the lin-4 mutation, while equivalent splice site mutations in construct int_5 retained rescue activity. A detailed summary of the percentages of transgenic animals in each phenotypic category for each transgenic line is found in Supplemental Figures 1 and 3. The contrasting results between int_3sm and int_5sm may be due to the differences in intron location within the

A

(i) Intron sequence 5' GTaagtttaaacagttcggtactaactaaccatacatatttaaattttcAG 3'

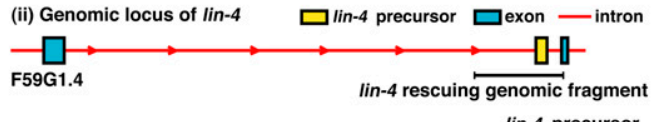

B

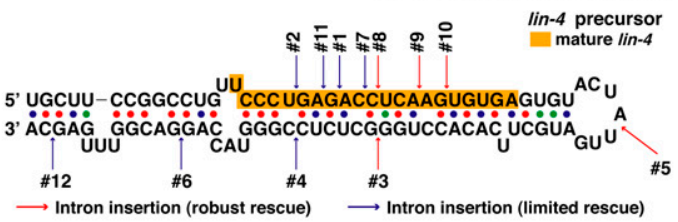

C

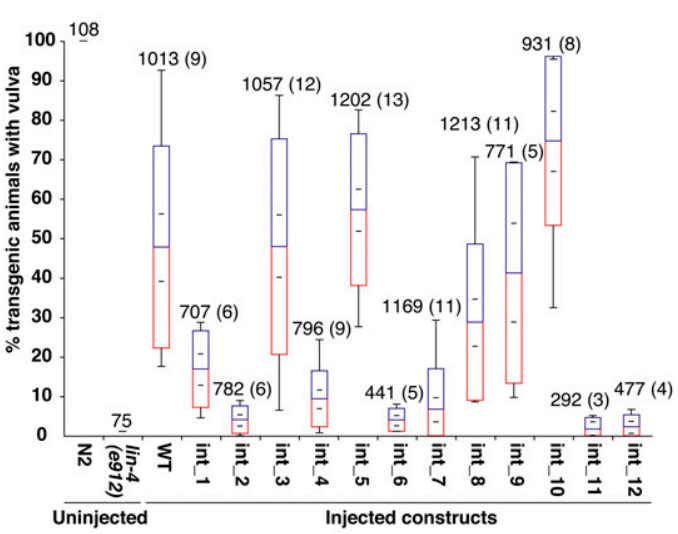

D

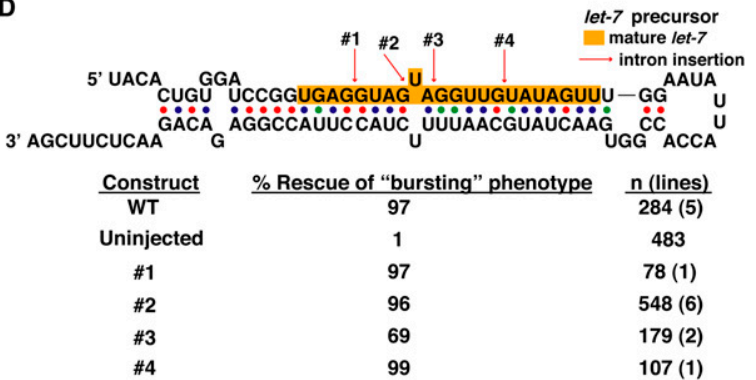

miRNA precursor; the intron in int_3 is located within the double-stranded stem, whereas the intron in int_5 is located in the loop region (Fig. 2B). These results suggest a flexibility in the loop region that may be absent in the regions destined to comprise the mature miRNA.

\section{Generation of 'wild-type' lin-4 miRNA from intron-containing lin-4 constructs}

We prepared small RNA libraries from transgenic lin-4(e912) lines for high-throughput sequencing using the Illumina platform. These libraries were prepared using the $5^{\prime}$ phosphate-dependent protocol to enrich for miRNAs (Pfeffer et al. 2005). The small RNA reads were aligned to both the wild-type lin-4 precursor and the corresponding intron-containing lin-4 precursor in order to capture all lin-4 variants.

As indicated in Figure 4A, both mature lin-4 and lin-4* were observed in four transgenic lines with robust rescue activities (int_5, int_5sm, int_8, and int_10), as well as in two transgenic lines with limited rescue activities (int_1 and int_11). We note that the more active inc-miR constructs (int_5, int_8, and int_10) produce mature lin-4 at levels in a range that is within several-fold of that observed in wild-type animals (Fig. 4A). Although measurement of absolute miRNA levels through RNA-seq is subject to sequence-specific variations in capture and sequencing efficiencies, a number of studies validates the use of differences in sequence count ratios in measuring relative miRNA levels as they change

Figure 2. In vivo function of inc-miR constructs. $(A)$ Key features used in creating intron-interrupted lin-4 constructs. (Panel i) Sequence of the 51-nt synthetic intron used is shown here, with the consensus splice donor (GT) and acceptor (AG) in red. (Panel ii) Relative position of the 695-nt lin-4(+) genomic fragment (black line) to host gene F59G1.4 (above black line) is illustrated. The lin-4(+)containing genomic fragment overlaps with both the intron (red line) and exon (blue box) of F59G1.4. (B) Sites of intron insertion in the lin-4 precursor sequence. The lin-4 precursor sequence is shown, with the mature lin-4 sequence highlighted in orange. Colored arrows indicate sites of intron insertion within the precursor sequence; different colors indicate different levels of lin-4 rescue activity (limited or robust) associated with each intron-interrupted lin-4 construct. (C) Percentages of transgenic animals with lin-4 rescue activity for intron-interrupted lin- 4 constructs. Percentages of transgenic animals with vulva (including egg-laying, pvl, and bursting phenotypes) for each intron-interrupted lin-4 construct are represented by a series of box plots. Multiple transgenic lines were assayed for each intron-interrupted lin-4 construct. The average percentage of transgenic animals displaying specific lin-4 rescue activity across all transgenic lines for each construct is represented by the band in the middle of the box plot (intersection of blue and red boxes). The top and bottom of each box plot represent the standard deviation between the different transgenic lines assayed. The black dashes within each box plot represent the standard error of mean for that construct. Maximum and minimum percentages of transgenic animals with specific lin-4 rescue activity observed for each construct are shown by the top and bottom whiskers of the box plot. The number above each box plot is the total number of transgenic animals assayed for each construct, and the number in parentheses is the number of transgenic lines assayed for each construct. The percentages for uninjected control animals [N2 and lin-4(e912)] are represented by single black lines. (D) Intron-containing let-7 constructs functionally rescue the let-7(n2853) mutation. The let-7 precursor sequence is shown, with mature let-7 sequence highlighted in orange. Red arrows indicate sites of intron insertion within the precursor sequence. The table summarizes the percentages of transgenic animals showing rescue of vulval bursting phenotype for each intron-interrupted let- 7 construct at $25^{\circ} \mathrm{C}$. 
A
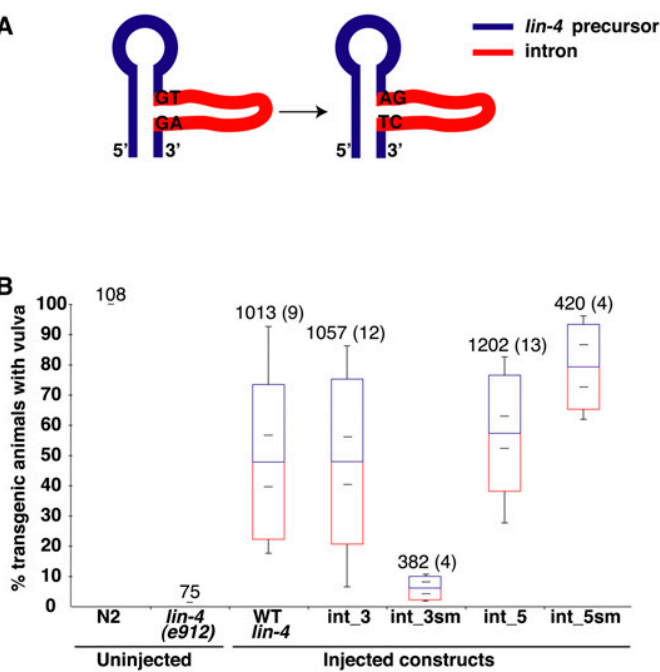

Figure 3. Splicing is required for lin-4 rescue activity of introninterrupted lin-4 constructs. (A) Mutations at consensus splice sites. The consensus splice donor (GT) and acceptor (AG) sequences in the introns (red) of two intron-interrupted lin-4 constructs (int 3 and int 5) were mutated to AG and CT, respectively. The site of intron insertion remained unchanged. (B) Percentages of transgenic animals with lin- 4 rescue activity for intron-interrupted lin-4 constructs with splice site mutations. Percentages of transgenic animals with vulva (including egg-laying, pvl, and bursting phenotypes) for each splice site mutation construct (int $3 \mathrm{sm}$ and int $5 \mathrm{sm}$ ) are represented by a series of box plots. These splice site mutation constructs are compared against their parent constructs (int_3 and int_5) and control uninjected animals. Data for the parent constructs and control animals were taken from previous experiments (Fig. 2C). Multiple transgenic lines were assayed for each construct. The average percentage of transgenic animals displaying specific lin- 4 rescue activity across all transgenic lines for each construct is represented by the band in the middle of the box plot (intersection of blue and red boxes). The top and bottom of each box plot represent the standard deviation between the different transgenic lines assayed. The black dashes within each box plot represent the standard error of mean for that construct. Maximum and minimum percentages of transgenic animals with specific lin- 4 rescue activity observed for each construct are shown by the top and bottom whiskers of the box plot. The number above each box plot is the total number of transgenic animals assayed for each construct, and the number in parentheses is the number of transgenic lines assayed for each construct. The percentages for uninjected control animals [N2 and lin-4(e912)] are represented by single black lines.

in different conditions (Lui et al. 2007; Marioni et al. 2008; Subramanian et al. 2008; Xu et al. 2010). Thus, sequence counts for lin- 4 relative to total miRNA counts provide a metric for relative changes in lin-4 abundance between different transgenic strains. The fact that some mature lin-4 and lin-4* were captured from transgenic lines with limited rescue activities from int_1 and int_11 (Fig. 4A) leaves either insufficient expression, inefficient splicing, or inefficient engagement of the resulting small RNA in the miRNA machinery as possible explanations of limited lin-4 rescue activities in these intron-interrupted lin-4 constructs. An analysis of start and end positions of captured lin-4 reads shows that the majority of lin-4 reads start and end at similar positions as wildtype mature lin-4 captured from N2 animals (Fig. 4B; Supplemental Fig. 4). Together, these results demonstrate that intron-containing lin-4 constructs were accurately spliced to generate wild-type lin-4.
Consistent with the retention of rescue activity by int_5sm (Fig. 3B), sequencing of small RNA populations in transgenic int_5sm lin-4(e912) animals yielded wildtype lin-4 and lin-4* (Fig. 4A). The majority of lin-4 reads start and end at similar positions as wild-type mature lin-4 captured from N2 animals (Fig. 4C). We did not capture other small RNA sequences that originate from the rest of unspliced int_5sm precursor, suggesting that the observed robust rescue is not likely a result of random "dicing" of unspliced int_5sm precursor to give sufficient levels of functional lin-4. Another possible explanation would be that mature wild-type lin-4 was stabilized, while other Dicer products of the int_5sm precursor were rapidly degraded.

\begin{tabular}{|c|c|c|c|c|}
\hline$A_{\text {Strain }}$ & $\underline{\text { lin-4 }}$ & $\underline{\text { lin-4* }}$ & \% lin-4 & $\underline{\text { Sequenced reads }}$ \\
\hline N2 & 18,838 & 18 & 0.85 & $3,423,171$ \\
\hline lin-4 (e912) & 0 & 0 & 0 & $4,133,073$ \\
\hline Int_1 & 3,304 & 42 & 0.27 & $2,447,053$ \\
\hline Int_5 & 27,936 & 16 & 0.35 & $9,668,616$ \\
\hline Int $5 \mathrm{sm}$ & 7,392 & 186 & 0.17 & $5,264,110$ \\
\hline Int 88 & 4,040 & 35 & 0.30 & $2,238,279$ \\
\hline Int_10 & 3,230 & 20 & 0.99 & $1,307,886$ \\
\hline Int_11 & 527 & 3 & 0.04 & $2,455,297$ \\
\hline
\end{tabular}
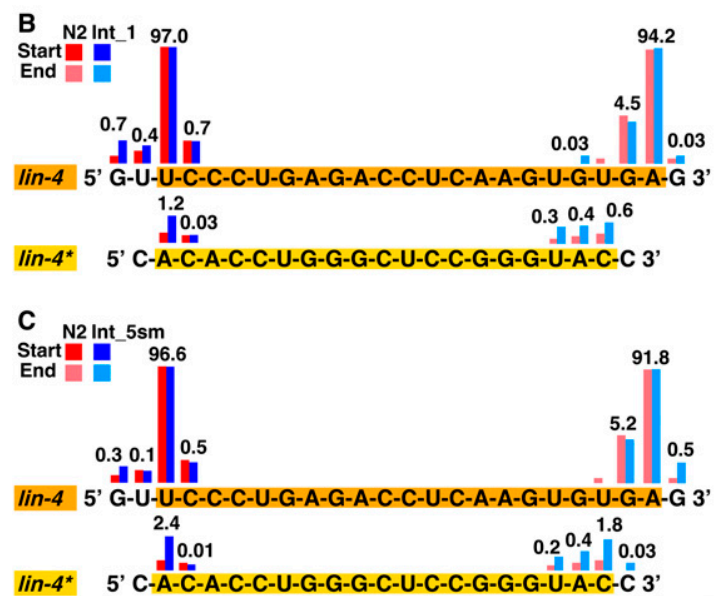

Figure 4. Generation of "wild-type" lin-4 miRNA from introncontaining lin-4 constructs. (A) Wild-type lin-4 and lin-4* are captured from lines expressing intron-interrupted lin- 4 constructs. The numbers of lin- 4 and lin- $4 *$ captured from high-throughput sequencing of small RNA populations from several transgenic lines are shown. The percentages shown are percentages of captured lin-4 against total captured miRNAs. Start and end positions of captured lin-4 and lin- $4^{*}$ are illustrated in $B$ and $C$. Mature lin- 4 sequence is highlighted in orange (top) and lin- $4^{*}$ sequence is highlighted in yellow (bottom). The colored bar above each nucleotide represents the percentage of lin-4 and lin- $4^{*}$ reads over total lin-4 and lin- $4^{*}$ reads that start/end at that position. Percentages are shown as numbers above the bars; only percentages for transgenic lines are shown. The heights of the colored bars are made directly proportional to $3 \sqrt{ }$ percentages to enable visualization of small values. The same N2 data set was used for all plots. (B) Start and end positions for lin-4 and lin- $4^{*}$ from transgenic line 183.3.1.1 (int_1) with limited levels of lin-4 rescue activity. Diagram shows small RNA productions from transgenic line 183.3.1.1 carrying int 1 construct with limited levels of lin-4 rescue activity. Both lin-4 and $\operatorname{lin}-4^{*}$ sequences were captured, with similar start and end positions to those of lin-4 and lin- $4^{*}$ captured from N2 strain. $(C)$ Start and end positions for lin-4 and lin- $4^{*}$ from transgenic rescued line 150.4.1.1 (int_5sm) carrying intron-interrupted lin-4 construct with splice site mutations. Diagram shows small RNA productions from transgenic rescued line 150.4.1.1 carrying int_5sm construct. Both lin-4 and lin- $4^{*}$ sequences were captured, with similar start and end positions to those of lin-4 and lin- $4^{*}$ captured from N2 strain. 


\section{In vivo function of intron-containing let-7 constructs}

To further assess the functionality of inc-miRs, introninterrupted versions of a different $C$. elegans miRNA, let-7, were constructed and assayed for phenotypic rescue of the let-7(n2853) mutation. The heterochronic miRNA let-7 is highly conserved across species (Pasquinelli et al. 2000) and is important for temporal development, especially during the larval-to-adult transition in C. elegans (Reinhart et al. 2000). The temperature-sensitive let-7(n2853) mutation causes adult lethality through vulval bursting at higher temperatures (Reinhart et al. 2000). Functional rescue of the bursting phenotype in transformed let-7(n2853) animals at nonpermissive temperatures $\left(25^{\circ} \mathrm{C}\right)$ allows us to test the functionality of intron-interrupted let-7 constructs. As shown in Figure $2 \mathrm{D}$, the four intron-interrupted let-7 constructs tested were able to rescue adult lethality associated with vulval bursting at $25^{\circ} \mathrm{C}$ in transgenic let-7(n2853) animals. These results demonstrate that the capacity of introninterrupted miRNAs to function in biological contexts is not limited to lin-4, suggesting instead that this capacity may be a general property of (at least) a substantial subset of miRNA effectors.

\section{Implications of functional inc-miRs}

In this study, we demonstrate that functional metazoan miRNAs can be processed from intron-interrupted precursors. This analysis highlights both the potential existence of spliced miRNAs in physiological systems and the potential utility of intron-interrupted miRNA transgene constructs as biotechnology tools. Since previous studies advancing sequence-based discovery of miRNAs have all required a contiguous alignment to the genome, intron-interrupted entities would have been missed (Ambros et al. 2003). The small size of mature miRNAs, combined with inherent variability in intron length, sequence, and structure, confounds any statistically and biologically definitive attempt to assign any given miRNA to a specific intron-interrupted genome segment. Nonetheless, our work argues that such entities may (and are perhaps likely to) exist. With the wealth of sequencing data coming from various high-throughput sequencing platforms, a significant portion of sequence reads are discarded as "unaligned." Although many different mechanisms may account for these sequence reads, the functionality of the inc-miR constructs indicates that unaligned sequences cannot simply be ignored. Together with the recent discovery of mirtrons /Okamura et al. 2007; Ruby et al. 2007), our observations illustrate the diversity in miRNA biogenic mechanisms and encourage consideration of all possibilities in the discovery of novel miRNAs.

\section{Materials and methods}

\section{Transgene-based expression of inc-miR constructs}

Intron-containing lin-4 constructs lin-4 DNA and marker plasmid (pC1\{pha-1(+)\}] (Granato et al. 1994) were mixed and introduced into populations carrying the lin-4-null mutation $e 912$, and transgenic populations were then assayed for their temporal progression phenotypes. We note that transgenic strains with wild-type lin- 4 are subject to differing degrees of partial rescue and mosaicism, with perfect rescue (full phenotypic rescue and egg-laying) observed in only a fraction of transgenic animals (Supplemental Fig. 1). Based on a titration of wild-type lin-4 plasmid pHZ018, we found optimal rescuing activity in a range encompassing a ratio of lin-4 construct to marker plasmid of 1:4 or 1:8. Each mutant construct was then assayed for rescue for both ratios. A complete description of the constructs, concentrations, and strains used in the transgenic experiments is provided in the Supplemental Material.

Intron-containing let-7 constructs let-7 DNA and marker plasmid (pPD117.01[pmec-7::gfp]) (Chalfie et al. 1994; Fire et al. 1998) were introduced into MT7626 [let-7(n2853)] (Reinhart et al. 2000), and resulting transgenic populations (animals with green fluorescent touch receptor neurons) were assayed for rescue of vulval bursting phenotype. A complete description of the constructs and concentrations used in the transgenic experiments is provided in the Supplemental Material.

\section{Splice site mutations of intron-containing lin-4 constructs}

Due to their robust lin-4 rescue activities and locations of introns outside the lin-4 mature sequence, the possibility that either int_3 or int_5 could have produced functional lin-4 without splicing was of particular interest. We mutated the splice sites of intron-interrupted lin-4 constructs int_3 and int_5 by substituting AG for GT at the splice donor site and CT for AG at the splice acceptor site (Fig. 3A), yielding constructs int_3sm (pHZ133) and int_5sm (pHZ136), respectively. Transgenic lines were derived in a similar manner by coinjection with pha-1(+) into PD7143 as described above and assayed for rescue of lin-4(e912) vulvaless phenotype.

\section{Preparation of small RNA libraries for Illumina sequencing}

Small RNA libraries were made from the following strains: PD1473 (line 183.3.1.1), PD1416 (line 140.2.2.1), PD1472 (line 150.4.1.1), PD1465 (line 174.1.3.1), PD1445 (line 179.1.4.1), PD7142 (line 25.1.3), N2, and lin-4(e912). A detailed description on library preparation is presented in the Supplemental Material.

\section{Sequence analysis of small RNA libraries}

Thirty-six-nucleotide reads were generated from the small RNA libraries using the Illumina Genome Analyzer system. The captured small RNA sequences, after removal of linker and adaptor sequences, were aligned using BLAT against a reference list of known C. elegans mature miRNA sequences downloaded from miRBase (http://www.mirbase.org). The number of captured small RNA sequences that perfectly matched mature lin-4 was obtained for each library. We also searched the small RNA sequence data for examples of aberrantly spliced products (i.e., for sequences that could have been derived from cryptic donor or acceptor sites within the transgene-produced precursor RNAs); no such aberrantly spliced small RNAs were observed in these analyses.

\section{Acknowledgments}

We thank Karen Artiles, Sam Gu, Ayelet Lamm, and Julia Pak for useful discussions. This work was supported by NIH grant R01GM37706. H.Z. was supported by $A^{\star}$ STAR, Singapore. J.M.M. was supported by NIH (T32HG00044) and the National Science Foundation Graduate Research Fellowship Program. The MT7626 strain was provided by the Caenorhabditis Genetics Center, which is funded by the NIH National Center for Research Resources (NCRR).

\section{References}

Alvarez-Saavedra E, Horvitz HR. 2010. Many families of C. elegans microRNAs are not essential for development or viability. Curr Biol 20: 367-373.

Ambros V, Lee RC. 2004. Identification of microRNAs and other tiny noncoding RNAs by cDNA cloning. Methods Mol Biol 265: 131-158.

Ambros V, Bartel B, DP Bartel, Burge CB, Carrington JC, Chen X, Dreyfuss G, Eddy SR, Griffiths-Jones S, Marshall M, et al. 2003. A uniform system for microRNA annotation. RNA 9: 277-279.

Blumenthal T, Steward K. 1997. Cis-splicing in worms. In C. elegans II (ed. DL Riddle et al.), pp. 117-145. Cold Spring Harbor Laboratory Press, Plainview, NY. 
Bushati N, Cohen SM. 2007. microRNA functions. Annu Rev Cell Dev Biol 23: 175-205.

Cai X, Hagedorn CH, Cullen BR. 2004. Human microRNAs are processed from capped, polyadenylated transcripts that can also function as mRNAs. RNA 10: 1957-1966.

Chalfie M, Horvitz HR, Sulston JE. 1981. Mutations that lead to reiterations in the cell lineages of C. elegans. Cell 24: 59-69.

Chalfie M, Tu Y, Euskirchen G, Ward WW, Prasher DC. 1994. Green fluorescent protein as a marker for gene expression. Science 263: 802805.

Fire A, Ahnn J, Kelly W, Harfe B, Kostas S, Hsieh J, Hsu M, Xu S. 1998 GFP applications in C. elegans. In GFP strategies and applications (ed. M Chalfie, S Kain), pp. 153-168. John Wiley and Sons, New York.

Flynt AS, Greimann JC, Chung W-J, Lima CD, Lai EC. 2010. MicroRNA biogenesis via splicing and exosome-mediated trimming in Drosophila. Mol Cell 38: 900-907.

Granato M, Schnabel H, Schnabel R. 1994. pha-1, a selectable marker for gene transfer in C. elegans. Nucleic Acids Res 22: 1762-1763.

Johnston RJ, Hobert O. 2003. A microRNA controlling left/right neuronal asymmetry in Caenorhabditis elegans. Nature 426: 845-849.

Kim Y-K, Kim VN. 2007. Processing of intronic microRNAs. EMBO J 26: $775-783$.

Kim VN, Han J, Siomi MC. 2009. Biogenesis of small RNAs in animals. Nat Rev Mol Cell Biol 10: 126-139.

Kloosterman WP, Steiner FA, Berezikov E, de Bruijn E, van de Belt J, Verheul M, Cuppen E, Plasterk RHA. 2006. Cloning and expression of new microRNAs from zebrafish. Nucleic Acids Res 34: 25582569.

Lagos-Quintana M, Rauhut R, Lendeckel W, Tuschl T. 2001. Identification of novel genes coding for small expressed RNAs. Science 294: 853-858.

Lau NC, Lim LP, Weinstein EG, Bartel DP. 2001. An abundant class of tiny RNAs with probable regulatory roles in Caenorhabditis elegans. Science 294: 858-862.

Lee RC, Ambros V. 2001. An extensive class of small RNAs in Caenorhabditis elegans. Science 294: 862-864.

Lee RC, Feinbaum RL, Ambros V. 1993. The C. elegans heterochronic gene lin-4 encodes small RNAs with antisense complementarity to lin-14. Cell 75: 843-854.

Lee Y, Kim M, Han J, Yeom K-H, Lee S, Baek SH, Kim VN. 2004. MicroRNA genes are transcribed by RNA polymerase II. EMBO I 23: 4051-4060.

Li J, Yang Z, Yu B, Liu J, Chen X. 2005. Methylation protects miRNAs and siRNAs from a 3 '-end uridylation activity in Arabidopsis. Curr Biol 15: 1501-1507.

Lu C, Jeong DH, Kulkarni K, Pillay M, Nobuta K, German R, Thatcher SR, Maher C, Zhang L,Ware D, et al. 2008. Genome-wide analysis for discovery of rice microRNAs reveals natural antisense microRNAs (nat-miRNAs). Proc Natl Acad Sci 105: 4951-4956.

Luciano DJ, Mirsky H, Vendetti NJ, Maas S. 2004. RNA editing of a miRNA precursor. RNA 10: 1174-1177.

Lui WO, Pourmand N, Patterson BK, Fire A. 2007. Patterns of known and novel small RNAs in human cervical cancer. Cancer Res 67: 60316043.

Maniar JM, Fire AZ. 2011. EGO-1, a C. elegans RdRP, modulates gene expression via production of mRNA-templated short antisense RNAs. Curr Biol 21: 449-459.

Marioni JC, Mason CE, Mane SM, Stephens M, Gilad Y. 2008. RNA-seq: an assessment of technical reproducibility and comparison with gene expression arrays. Genome Res 18: 1509-1517.

Mattick JS, Makunin IV. 2006. Non-coding RNA. Hum Mol Genet 15: R17-R29. doi: 10.1093/hmg/dd1046.

Miska EA, Alvarez-Saavedra E, Abbott AL, Lau NC, Hellman AB, McGonagle SM, Bartel DP, Ambros VR, Horvitz HR. 2007. Most Caenorhabditis elegans microRNAs are individually not essential for development or viability. PLoS Genet 3: e215. doi: 10.1371/journal. pgen.0030215

Okamura K, Hagen JW, Duan H, Tyler DM, Lai EC. 2007. The mirtron pathway generates microRNA-class regulatory RNAs in Drosophila. Cell 130: 89-100.

Pasquinelli AE, Reinhart BJ, Slack F, Martindale MQ, Kuroda MI, Maller B, Hayward DC, Ball EE, Degnan B, Müller P, et al. 2000. Conserva- tion of the sequence and temporal expression of let-7 heterochronic regulatory RNA. Nature 408: $86-89$.

Pfeffer S, Lagos-Quintana M, Tuschl T. 2005. Cloning of small RNA molecules. In Current protocols in molecular biology (ed. FM Ausubel), pp. 26.4.1-26.4.18. John Wiley and Sons, New York. doi: $10.1002 / 0471142727 . \mathrm{mb} 2604 \mathrm{~s} 72$.

Rajagopalan R, Vaucheret H, Trejo J, Bartel DP. 2006. A diverse and evolutionarily fluid set of microRNAs in Arabidopsis thaliana. Genes Dev 20: 3407-3425.

Reinhart BJ, Slack FJ, Basson M, Pasquinelli AE, Bettinger JC, Rougvie AE, Horvitz HR, Ruvkun G. 2000. The 21-nucleotide let-7 RNA regulates developmental timing in Caenorhabditis elegans. Nature 403: 901-906.

Ruby JG, Jan CH, Bartel DP. 2007. Intronic microRNA precursors that bypass Drosha processing. Nature 448: $83-86$.

Soller M. 2006. Pre-messenger RNA processing and its regulation: a genomic perspective. Cell Mol Life Sci 63: 796-819.

Subramanian S, Lui WO, Lee CH, Espinosa I, Nielsen TO, Heinrich MC, Corless CL, Fire AZ, van de Rijn M. 2008. MicroRNA expression signature of human sarcomas. Oncogene 27: 2015-2026.

Sunkar R, Girke T, Jain PK, Zhu JK. 2005. Cloning and characterization of microRNAs from rice. Plant Cell 17: 1397-1411.

Thieme CI, Gramzow L, Lobbes D, Theissen G. 2011. SplamiR-Prediction of spliced miRNAs in plants. Bioinformatics 27: 1215-1223.

Wightman B, Ha I, Ruvkun G. 1993. Posttranscriptional regulation of the heterochronic gene lin-14 by lin-4 mediates temporal pattern formation in C. elegans. Cell 75: 855-862.

Xu G, Wu J, Zhou L, Chen B, Sun Z, Zhao F, Tao Z. 2010. Characterization of the small RNA transcriptomes of androgen dependent and independent prostate cancer cell line by deep sequencing. PLOS ONE 5: e15519. doi: 10.1371/journal.pone.0015519. 


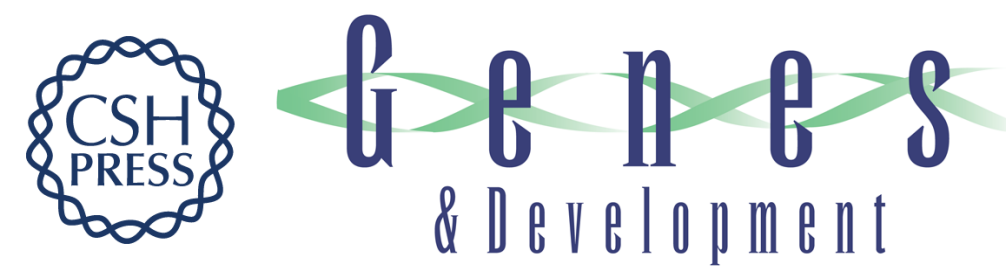

\section{'Inc-miRs': functional intron-interrupted miRNA genes}

Huibin Zhang, Jay M. Maniar and Andrew Z. Fire

Genes Dev. 2011, 25:

Access the most recent version at doi:10.1101/gad.2058711

Supplemental
Material http://genesdev.cshlp.org/content/suppl/2011/08/09/25.15.1589.DC1

References This article cites 37 articles, 13 of which can be accessed free at: http://genesdev.cshlp.org/content/25/15/1589.full.html\#ref-list-1

License

Email Alerting Receive free email alerts when new articles cite this article - sign up in the box at the top Service right corner of the article or click here.

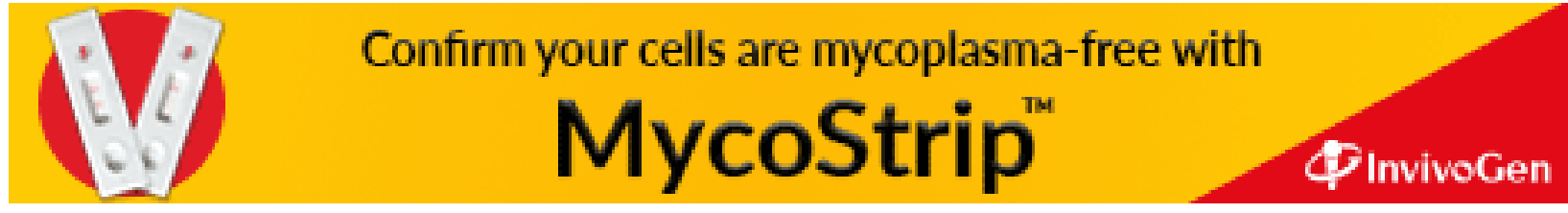

